\title{
Correction to: PRMT6 physically associates with nuclear factor Y to regulate photoperiodic flowering in Arabidopsis
}

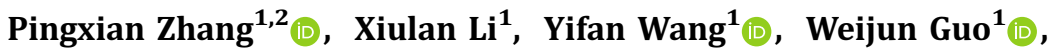 \\ Sadaruddin Chachar ${ }^{1}$, Adeel Riaz ${ }^{1}$, Yuke Geng ${ }^{3}$, Xiaofeng Gu¹ ${ }^{1}$, \\ Liwen Yang ${ }^{1 \bowtie}$ \\ ${ }^{1}$ Biotechnology Research Institute, Chinese Academy of Agricultural Science, Beijing 100081, China \\ 2 College of Life Science and Technology, Huazhong Agricultural University, Wuhan 430070, Hubei, China \\ 3 College of Life and Environmental Sciences, Minzu University of China, Beijing 100081, China
}

Published online: 24 December 2021

\section{Correction to: aBIOTECH \\ https://doi.org/10.1007/s42994-021-00065-y}

In this article Figs. 2 and 3 were wrongly numbered; Fig. 2 should have been Fig. 3 and vice versa as shown below. Moreover, the Fig. 3 indications have been revised as shown: the sentence "Next, two transfer DNA (T-DNA) insertion single- mutant prmt6-1 (Sail_385_ A06) and prmt6-2 (Salk_151679C) (Figs. 2A; S3a)..." has been revised as "Next,two transfer DNA (T-DNA) insertion single- mutant prmt6-1 (Sail_385_A06) and prmt6-2 (Salk_151679C) (Figs. 3A; S3a)...; And the sentence "...was significantly more than that of $n f-y c 3 ; 4 ; 9$ (Fig. 2B, D)..." has been revised as "...was significantly more than that of $n f-y c 3 ; 4 ; 9$ (Fig. 3B, D)...; And the sentence "...lower than that of the $n f-y c 3 ; 4 ; 9$ triple mutant line at ZT16 under LDs (Fig. 2E)..." has been revised as "...lower than that of the $n f-y c 3 ; 4 ; 9$ triple mutant line at ZT16 under LDs (Fig. 3E)...." The original article has been corrected.

The original article can be found online at https://doi.org/10. 1007/s42994-021-00065-y.

$\bowtie$ Correspondence: yangliwen@caas.cn (L. Yang) 


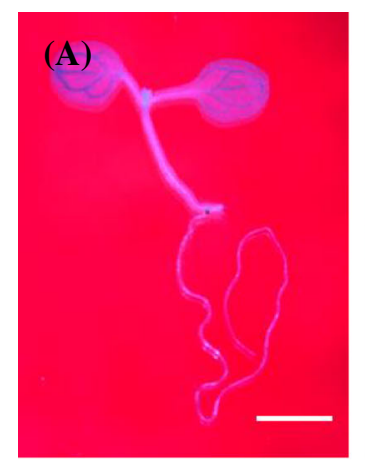

(B)

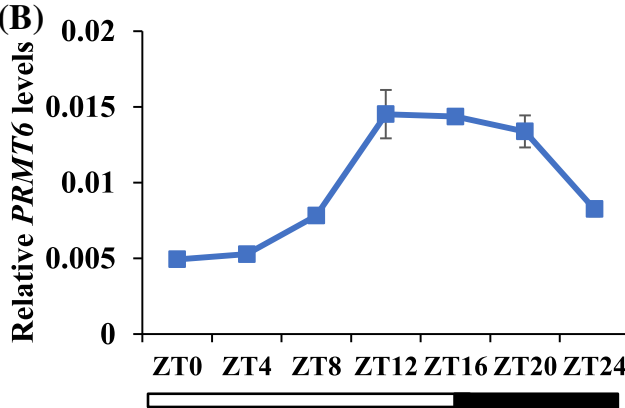

(C)

$$
\text { ZT(h) }
$$
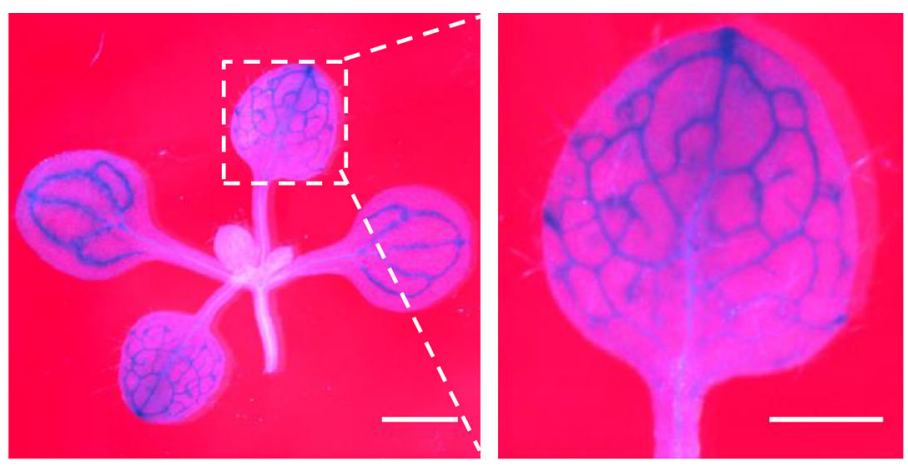

(D)

\section{Anti-PRMT6-FLAG}

\section{Anti-H3 control}

\section{Loading control}
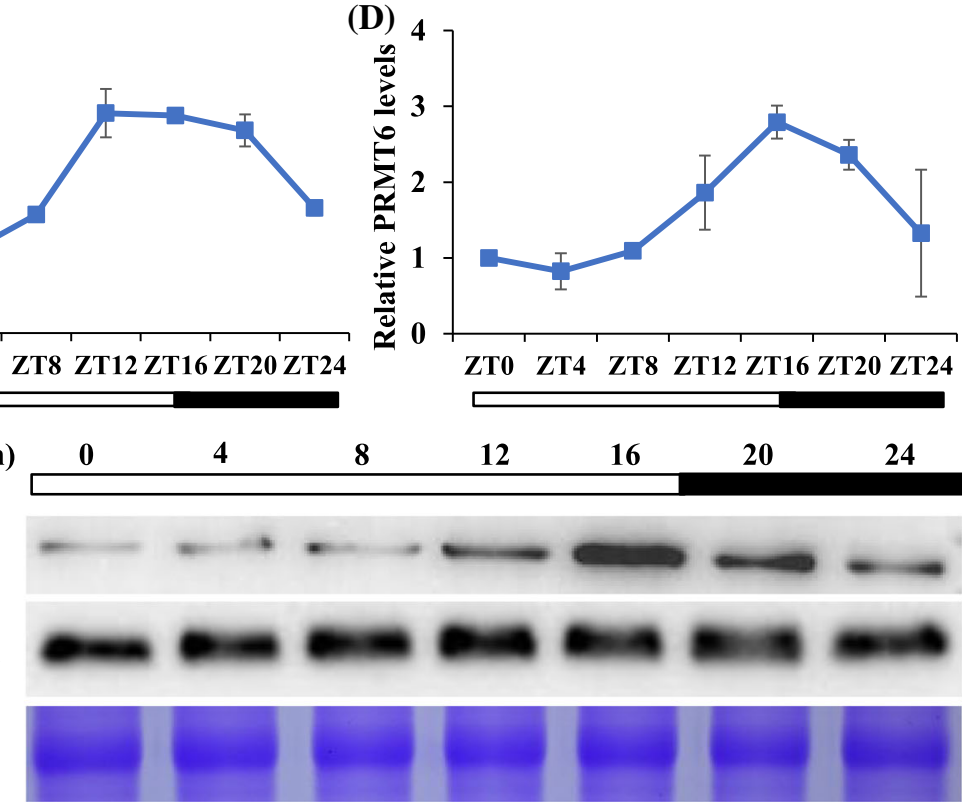

Fig. 2 PRMT6 diurnally expressed at dusk in the vascular bundle cells under LDs. A Spatial expression patterns of PRMT6-GUS in 5-dayold seedlings, and 10-day-old seedlings of aerial part and cotyledon. Plants were stained for $6 \mathrm{~h}$. Scale bars $=1 \mathrm{~mm}$. B The relative transcription level of PRMT6 in 10-day-old Col seedlings under LDs. The transcription levels were normalized to UBQ10, and relative fold changes to Zeitgeber time 0 (ZT0) are presented. Bars indicate s.d. of triplicate measurements. White and dark bars below the -axis indicate light and dark periods, respectively. C, D The exprxession levels of PRMT6-FLAG protein over a 24-h LD cycle examined by western blotting. Total proteins loaded in SDS-PAGE gels were stained with Coomassie Blue, antibody or the relative PRMT6-FLAG protein levels were normalized to H3 by the ImageJ program (D). The error bars indicate the s.d. measurements 
(A)

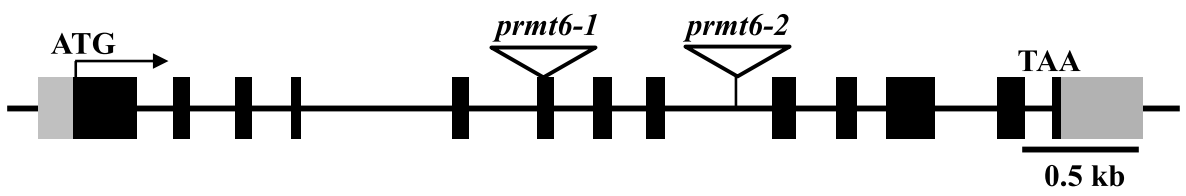

(B)

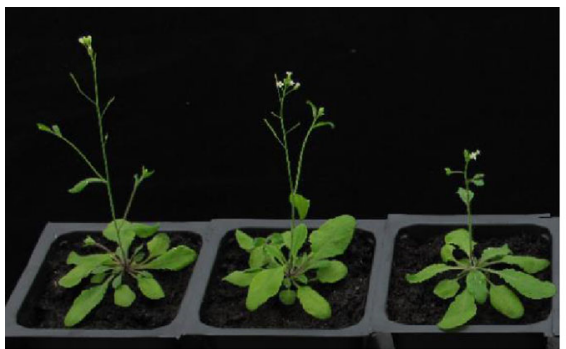

Col

prmt6-2 prmt6-1

(C)

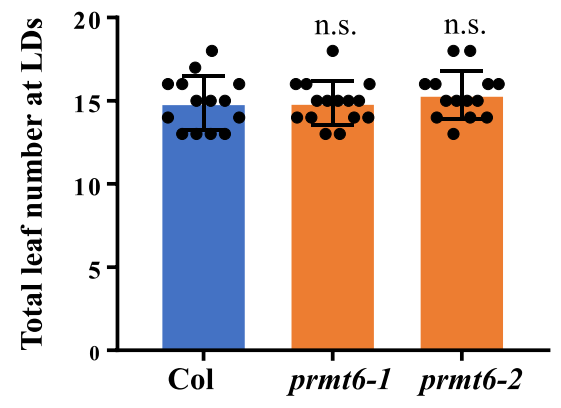

(E)

E)

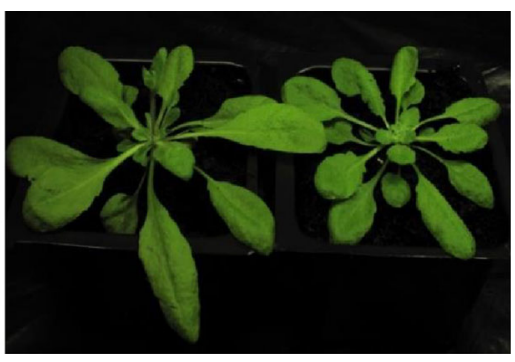

nf-yc3;4;9 prmt6-1;nf-yc3;4;9

(D)

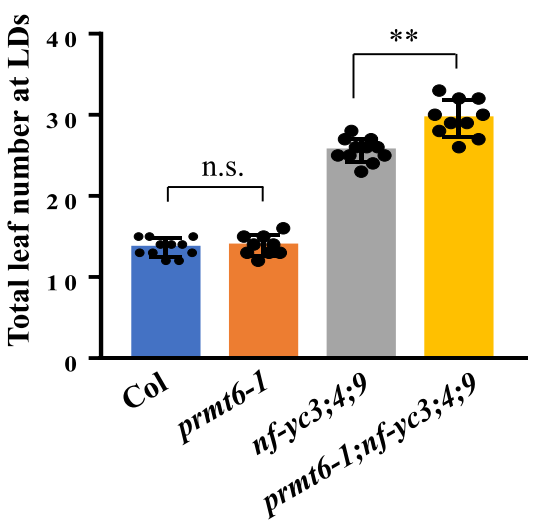

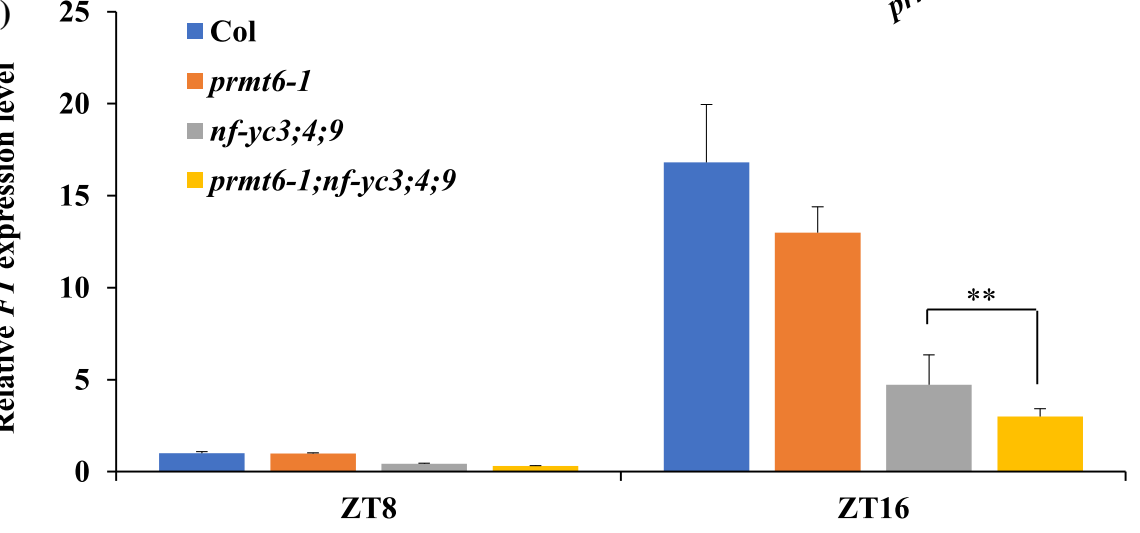

Fig. 3 Loss of PRMT6 function delays the floral transition of $n f-y c 3 ; 4 ; 9$ by decreasing the $F T$ expression under LDs. A Gene structure of PRMT6. Exons and 5' untranslated region (UTR) or 3' UTR are represented by black boxes and gray boxes, and arrows indicate transcription start sites (TSS); the T-DNA insertion sites of two lines are indicated with triangles. B Phenotype of Col, prmt6-1, prmt6-2, nf$y c 3 ; 4 ; 9$, and prmt6-1;nf-yc3;4;9 mutants grown in LDs. C Flowering times of the indicated genotypes grown in LDs. More than ten plants for each line were scored; bars indicated for standard deviation (s.d.); n.s. indicated non-significant difference. D Flowering times of the indicated lines grown in LDs. More than ten plants for each line were scored; bars indicated for s.d.; n.s. indicated non-significant difference; Double asterisks indicated statistically significant differences in the means between the indicated genotypes, as revealed by two-tailed Student's $t$ test $\left(^{* *} p<0.01\right)$. E Relative $F T$ transcript levels in the seedlings of the indicated genotypes grown in LDs at ZT8 and ZT16. The transcript levels were first normalized to that of UBQ10. Bars indicate the s.d. of triplicate measurements 
Open Access This article is licensed under a Creative Commons Attribution 4.0 International License, which permits use, sharing, adaptation, distribution and reproduction in any medium or format, as long as you give appropriate credit to the original author(s) and the source, provide a link to the Creative Commons licence, and indicate if changes were made. The images or other third party material in this article are included in the article's
Creative Commons licence, unless indicated otherwise in a credit line to the material. If material is not included in the article's Creative Commons licence and your intended use is not permitted by statutory regulation or exceeds the permitted use, you will need to obtain permission directly from the copyright holder. To view a copy of this licence, visit http://creativecommons.org/ licenses/by/4.0/. 